\title{
NaV1.1 and NaV1.6 selective compounds reduce the behavior phenotype in a novel zebrafish model for Dravet Syndrome
}

Wout J. Weuring ${ }^{1}$, Sakshi Singh ${ }^{1}$, Linda Volkers², Martin Rook ${ }^{3}$, Ruben H. van 't Slot',

Marjolein Bosma ${ }^{1}$, Marco Inserra ${ }^{4}$, Irina Vetter ${ }^{4}$, Nanda M. Verhoeven-Duif ${ }^{1}$, Kees P.J. Braun $^{5}$, Mirko Rivara ${ }^{6}$, Bobby P. C. Koeleman ${ }^{1}$

\section{Affiliations}

${ }^{1}$ Department of Genetics, University Medical Centre Utrecht, the Netherlands

${ }^{2}$ Department of Cardiology, Laboratory of Experimental Cardiology, University Medical Centre Leiden, the Netherlands

${ }^{3}$ Department of Medical Physiology, University Medical Centre Utrecht, the Netherlands ${ }^{4}$ Centre for Pain Research \& School of Pharmacy, University of Queensland, Australia.

${ }^{5}$ Department of Neurology, University Medical Centre Utrecht, the Netherlands

${ }^{6}$ Food and Drug department, University of Parma, Italy

\section{Correspondence to}

B. P. C. Koeleman

Department of Genetics

Centre of Molecular Medicine (CMM)

University Medical Centre (UMC)

STR.1.305, Lundlaan 6

3584 CG, Utrecht

The Netherlands

Email: b.p.c.koeleman@umcutrecht.nl

Phone: $+31(0) 887568116$

Fax: +31887567984

Keywords: Nav1.1, Nav1.6, SCN1A, SCN8A, GABA, VGSC

Number of Text pages: 15 Words: 5016 References: 39 Figures: 6 (+7 Supp.) 


\begin{abstract}
Dravet syndrome is caused by dominant loss-of-function mutations in SCN1A which cause reduced activity of Nav1.1 leading to lack of neuronal inhibition. On the other hand, gain-of-function mutations in SCN8A can lead to a severe epileptic encephalopathy subtype by over activating $\mathrm{Na}_{\mathrm{V}} 1.6$ channels. These observations suggest that Nav1.1 and Nav1.6 represent two opposing sides of the neuronal balance between inhibition and activation. Here, we hypothesize that Dravet syndrome may be treated by either enhancing Nav1.1 or reducing Nav1.6 activity. To test this hypothesis we generated and characterized a novel DS zebrafish model and tested new compounds that selectively activate or inhibit the human $\mathrm{Na}_{\mathrm{V}} 1.1$ or $\mathrm{Na}_{\mathrm{V}} 1.6$ channel respectively. We used CRISPR/Cas 9 to generate two separate Scn1Lab knockout lines as an alternative to previous knock-down models.
\end{abstract} Using an optimized locomotor assay, spontaneous burst movements were detected that were unique to Scn1Lab knockouts and disappear when introducing human SCN1A mRNA. Besides the behavioral phenotype, Scn1Lab knockouts show sudden, electrical discharges in the brain that indicate epileptic seizures in zebrafish. Scn1Lab knockouts showed increased sensitivity to the convulsant pentylenetetrazole and a reduction in whole organism GABA levels. Drug screenings further validated a Dravet syndrome phenotype. We tested the $\mathrm{Na}_{\mathrm{V}} 1.1$ activator AA43279 and our newly synthesized $\mathrm{Na}_{\mathrm{V}} 1.6$ inhibitors MV1369 and MV1312 in the Scn1Lab knockouts. Both type of compounds significantly reduced the number of burst movements. Our results show that selective inhibition of $\mathrm{Na}_{\mathrm{V}} 1.6$ could be just as efficient as selective activation of $\mathrm{Na}_{\mathrm{V}} 1.1$ and these approaches could prove to be novel potential treatment strategies for Dravet syndrome and other (genetic) epilepsies. Compounds tested in zebrafish however, should always be further validated in other model systems, preferably human derived.

\title{
Introduction
}

Dravet syndrome (DS), previously known as severe myoclonic epilepsy of infancy (SMEI), is a severe form of epilepsy for which current medication strategies remain largely inefficient. Promising new drugs that act on the serotonin pathway such as Fenfluramine (FA), show 
efficacy in reducing seizures in $50 \%$ to $90 \%$ of the patients ${ }^{[1]}$. However, drug side effects may still limit their use, underscoring the need for further drug discovery.

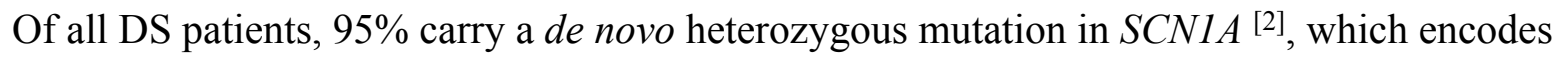
the pore forming $\alpha$-subunit of neuronal voltage gated sodium channel (VGSC) type 1 $\left(\mathrm{Na}_{\vee} 1.1\right) . \mathrm{Na}_{\mathrm{V}} 1.1$ ion channels are the primary $\mathrm{Na}^{+}$channels in GABAergic interneurons ${ }^{[3]}[4]$ and induce the fast depolarization of neuronal membranes during action potential initiation. The majority of SCN1A mutations in DS are loss-of-function (LOF) mutations resulting in dysfunctional $\mathrm{Na}_{\mathrm{V}} 1.1$ channels, or reduced $\mathrm{Na}_{\mathrm{V}} 1.1$ expression ${ }^{[5]}{ }^{[6]}$. Consequently, excitability and action potential amplitude of interneurons are attenuated leading to reduced GABA release ${ }^{[40]}$.

Another brain VGSC subtype, $\mathrm{Na}_{\mathrm{V}} 1.6$ is one of the two main sodium channels expressed in pyramidal neurons, which are responsible for excitatory signals via glutamate excretion ${ }^{[7]}$. $S C N 8 A$, which encodes the $\mathrm{Na}_{\mathrm{V}} 1.6 \alpha$-subunit is also related to epilepsy and approximately 100 mutations have been reported in patients with severe Early Infantile Epileptic Encephalopathy subtype 13 (EIEE13). Unlike the clear LOF mutations in SCN1A, the majority of the functionally tested $S C N 8 A$ mutations result in gain-of-function (GOF) of $\mathrm{Na}_{\mathrm{V}} 1.6^{[8]}$. GOF mutations in $\mathrm{Na}_{\mathrm{V}} 1.6$ cause channel hyperactivity due to augmented excitability and firing rates of pyramidal cells concurrent with an increase in glutamate release.

This disease mechanism is reflected by the therapeutic response of VGSC blockers. Various clinical reports have shown that $S C N 8 A$-related epilepsy patients benefit from VGSC blockers [9] [10], contrasting their inefficacy, or even detrimental effects in DS ${ }^{[11]}$. These observations indicate that $\mathrm{Na}_{\mathrm{V}} 1.1$ and $\mathrm{Na}_{\mathrm{V}} 1.6$ represent two opposing sides of the neuronal balance between inhibition and activation. We hypothesize that LOF mutations in SCN1A have a major negative effect on neuronal inhibition via hypo- activity of inhibitory interneurons, 
shifting the balance to neuronal hyperactivity. In contrast, GOF SCN8A mutations cause increased activity of excitatory pyramidal neurons, also shifting the VGSC-related balance towards neuronal hyperactivity. This model suggests that either selective activation of $\mathrm{Na}_{\mathrm{V}} 1.1$ or selective inhibition of $\mathrm{Na}_{\mathrm{V}} 1.6$ could be a therapeutic approach in the treatment of both DS and $S C N 8 A$-related epilepsy.

The therapeutic effect of $\mathrm{Na}_{\mathrm{V}} 1.1$ activation was previously shown in DS mice using spider venom peptide $\mathrm{Hm} 1 \mathrm{a}(\mathrm{HmTx} 1)$ that led to a reduction of seizures and mortality ${ }^{[12]}$. In another study using a different mouse strain, Hmla was found to be lethal at picomolar doses within two hours ${ }^{[13]}$, indicating that safety and administration needs to be further studied. Another $\mathrm{Na}_{\mathrm{V}} 1.1$ activator is the chemically synthesized small molecule AA43279, which showed anticonvulsant properties in-vivo but has not been tested in an animal model for DS. In comparison with $\mathrm{Hm1a}$, AA43279 is less selective for $\mathrm{Na}_{\mathrm{V}} 1.1$, indicating it could activate other $\mathrm{Na}_{\mathrm{V}}$ subtypes at lower concentrations. Nevertheless, AA43279 did not show lethality or sedative and ataxic side-effects at a concentration of $300 \mathrm{mg} / \mathrm{kg}$ in mice ${ }^{[15]}$. Inhibition of $\mathrm{Na}_{\mathrm{V}} 1.6$ in DS was previously mimicked by introducing an $S C N 8 A$ missense mutation in DS mice, which reduced seizure susceptibility and increased their lifespan ${ }^{[14]}$. Compounds that block $\mathrm{Na}_{\mathrm{V}} 1.6$ selectively have not been published to date.

To test if both $\mathrm{Na}_{\mathrm{V}} 1.1$ agonists and $\mathrm{Na}_{\mathrm{V}} 1.6$ antagonists could be beneficial in the treatment of DS we generated a novel DS zebrafish model by knocking out the Scn1Lab gene using CRISPR/Cas9. In humans, most of the mutations that cause DS are severe truncating mutations, while mild missense mutations are observed in patients with a milder epileptic phenotype ${ }^{[41]}$. To mimic the genetic architecture of DS in human patients as best possible, the animal model should display a 50\% haploinsufficiency of SCN1A. Zebrafish likely carry two orthologues for the human SCN1A gene; Scn1Laa and Scn1Lab. While the expression of these genes does not overlap at embryonic- but only at larval stages ${ }^{[18][25]}$, they have a shared 
functional role in epilepsy ${ }^{[20]}$. Since 2010, three other Scn1Lab zebrafish models have been introduced, generated by N-ethyl-N-nitrosourea (ENU) mutagenesis ${ }^{[18]}{ }^{[19]}$ or morpholino antisense oligomers (MO) ${ }^{[17]}$. All three display an epileptic phenotype that includes hyperactivity and epileptic spikes recorded from the brain. Nevertheless, CRISPR/Cas9 is currently the most efficient technique to specifically disrupt the gene that is targeted, unlike ENU mutagenesis ${ }^{[21]}$, and acts on the DNA rather than protein as with MO based approaches [23][24]. To mimic DS in human patients, we generated two zebrafish models with different truncating mutations in ScnlLab using CRISPR/Cas9.

The epileptic phenotype and drug response in zebrafish larvae can be measured by quantifying high velocity burst movements which are indicative of epileptic seizures in fish. The effect of anti-epileptic drugs on this unique behavior phenotype was found to be well correlated with actual epileptic spikes recorded from the zebrafish brain ${ }^{[33]}$. For this reason, we used the local field potential set-up in combination with a behavior essay to establish the initial phenotype in ScnlLab knockouts, but used the locomotor assay as a single read-out on the drugs tested. After further molecular validation of the animal model, we tested $\mathrm{Na}_{\mathrm{V}} 1.1$ agonist AA43279 and synthesized and two novel $\mathrm{Na}_{\mathrm{V}} 1.6$ channel antagonists, MV1369 and MV1312. Our results show that selective targeting of $\mathrm{Na}_{\mathrm{V}} 1.1$ or $\mathrm{Na}_{\mathrm{V}} 1.6$ ion channels reduced seizure activity in ScnlLab knockout zebrafish, indicating a restoration in neuronal signaling.

\section{Results}

\section{Generation of Scn1Lab knockout zebrafish}

Heterozygous and homozygous Scn1Lab knockout zebrafish were generated using CRISPR/Cas9. A 13 bp deletion was created in Scn1Lab exon 10, generating a STOP codon on position 474, truncating the Scn1Lab protein (S2a-c Figure). From here on, Scn1Lab knockout indicates 5 days post fertilization (dpf) larvae carrying the homozygous $13 \mathrm{bp}$ 
deletion in Scn1Lab. A second allele, carrying a 5 bp deletion in exon 10, leading to a STOP codon on position 487 was generated in parallel to confirm the knockout phenotype (S2b

Figure). To validate Cas9' specificity in DNA editing, potential off-target regions were sequenced. No off-target editing of Cas9 was observed (S3 Figure). To verify the presence of the genomic deletion at transcription level, cDNA was sequenced, resulting in detection of the deletion (S4 Figure). Scn1Lab knockouts showed a similar morphological phenotype as observed in previous ScnlLab knock-down models [17] [18] [19] including hyper-pigmentation and the absence of an inflated swim bladder (Figure 1a). Heterozygous knockout ScnlLab larvae showed no apparent morphological difference compared to wildtype zebrafish (Figure 1b-c). As a control for the morphological differences of ScnLab knockouts, we generated nisb-WT control zebrafish ${ }^{[17]}$ that lack an inflated swim bladder (S5 Figure).

\section{Epileptic phenotype of Scn1Lab knockouts}

Scn1Lab knockouts showed behavior comparable to previously described Scn1Lab knockdown models ${ }^{[17][18][19]}$ including hyperactivity and high velocity burst movements (S6 video). Using optimized parameters, these specific, high velocity $(>50 \mathrm{~mm} / \mathrm{s})$ burst movements were separated from regular locomotor data to yield a burst movement assay. Scn1Lab knockouts showed a significantly higher number of spontaneous burst movements (Figure 2a). Using the nisb-WT control zebrafish, burst movements were found to be unique to Scn 1Lab knockouts and not caused by the absence of an inflated swim bladder (Figure 2b). No spontaneous burst movements were observed in heterozygous knockout Scn1Lab larvae (Figure 2a). Using a local field potential (LFP) configuration for zebrafish embryos ${ }^{[26]}$, abnormal brain activity was observed in Scn1Lab knockouts including spontaneous, singleand poly spiking electrical discharges. The LFP patterns recorded from Scn1Lab knockouts resembles those observed in previous Scn1Lab knock-down zebrafish models [17] [18] (Figure 2f). 


\section{Partial rescue of Scn1Lab knockout burst movements by human SCN1A}

Scn1Lab is believed to be one of the two functional orthologues for human SCN1A, therefore we tested whether the spontaneous burst movement phenotype of ScnlLab knockouts can be rescued by the introduction of human $S C N 1 A$ in our model. Human $S C N 1 A$ mRNA or cDNA was injected in one-cell stage Scn1Lab knockout embryos leading to a partial rescue of burst movements. (Figure 2c).

\section{GABA reduction and sensitivity to pentylenetetrazole (PTZ) in Scn1Lab knockouts}

To see if the GABAergic tone is disturbed in our Scn1Lab knockouts, levels of free GABA were quantified in whole organism by Mass-spectrometry. Scn1Lab knockouts showed a statistically significant reduction of GABA (Figure 2d). Thus, as Scn1Lab knockouts are GABA deficient, they might be more susceptible to convulsing agents that act on the GABAergic inhibitory pathway. PTZ, a GABA antagonist frequently used as convulsant in animal studies was applied to Scn1Lab knockouts. Heterozygous and homozygous Scn1Lab knockouts showed a statistically significant increase in burst movements when exposed to 5mM PTZ, compared to wildtype zebrafish (Figure 2e).

\section{Pharmacological validation confirms a DS phenotype}

Traditional VGSC blockers are known to be inefficient in SCN1A related epilepsies and can even sometimes aggravate seizures in Dravet syndrome, likely due to their lack of $\mathrm{Na}_{\mathrm{V}}$ subtype specificity. To test if VGSC blockers alter the burst movement phenotype in our ScnlLab knockouts, the anti-epileptic drugs (AEDs) Phenytoin (PTH) and Carbamazepine (CBZ) were applied using a short or long drug exposure time. No reduction in burst movements was observed in the ScnlLab knockouts (Figure 3a, b) when exposed to PTH or CBZ, confirming the inefficacy of VGSC blockers in DS. Next we tested the anti-seizure effects of GABA enhancing drugs Valproic acid (sodium valproate, VPA) and Stiripentol (STP), which are effective treatments for recurrent seizures in DS. Seizure-like burst 
movements were significantly reduced when Scn1Lab knockouts were exposed to STP

(Figure 3d). Interestingly, VPA showed an effect only after long exposure, short exposure did not result in burst movement reduction (Figure 3c).

\section{Partial seizure reduction by serotonin pathway modulators}

Fenfluramine has previously been discovered in the Scn ILab morpholino knock-down model to be effective in reducing DS seizures ${ }^{[17]}$ and is now shown to be equally effective in our Scn1Lab knockout (Figure 3e). Clemizole (CLM), an antihistamine that can also bind to the serotonin receptor did not show a significant reduction of burst movements in the Scn ILab knockouts unlike a previous Scn1Lab knock-down model ${ }^{[18]}$. Toxicity of CLM was observed in the Scn 1Lab knockout long exposure groups, showing mortality and body malformations at previous established concentrations for a short exposure experiment ${ }^{[18]}$ (S7 Figure). When exposed to $50 \%$ of this dose, no toxicity was observed and no significant reduction in burst movements was observed (Figure 3f).

\section{Nav1.1 selective- but not general VGSC activators reduce seizures}

Wildtype zebrafish exposed for a short or long incubation time to the general VGSC activator Veratridine (VRT) developed burst movements, confirming the convulsing effects of VRT in healthy control animals (Figure 4a). Interestingly, ScnlLab knockouts revealed no additional increase, nor decrease of burst movements after being exposed to VRT (Figure 4a). When exposed to $\mathrm{Na}_{\mathrm{V}} 1.1$ selective activator AA43279, the number of burst movements in Scn1Lab knockouts was significantly decreased (Figure 4b). This effect was only observed in the short, but not in the long exposure group, confirming the rapid clearance of AA43279 that has been reported in mice ${ }^{[15]}$.

\section{Selectivity of MV1312 and MV1369 and efficacy in Scn1Lab knockouts}

From a range of unpublished VGSC blocking compounds, several were tested for human $\mathrm{Na}_{\vee} 1.6$ selectivity. MV1312 had a 5-6 fold selectivity of $\mathrm{Na}_{\mathrm{V}} 1.6$ over $\mathrm{Na}_{\mathrm{V}} 1.1-1.7$, but 
comparable blocking affinity for $\mathrm{Na}_{\mathrm{V}} 1.8$ (Figure 5a). $\mathrm{Na}_{\mathrm{V}} 1.8$ is a peripheral nervous system ion channel involved in the sensation of pain, and blockage could lead to anti-nociception and pain treatment. As the selectivity over $\mathrm{Na}_{\mathrm{V}} 1.1$ is most important, we chose compound MV1312 to be a suitable candidate for further evaluation in our DS animal model. When exposed to $5 \mu \mathrm{M}$ MV1312, the number of burst movements was statistically significant reduced (Figure 5b), indicating a restoration of neuronal signaling in the epileptic brain. Compound MV1369 showed less selectivity for $\mathrm{Na}_{\mathrm{V}} 1.6$, but did show selectivity over the CNS channel Na 1.2 (Figure 5c). Interestingly, when applied MV1369, the number of burst movements was also statistically significant reduced (Figure 5d). As the DNA and proteins differ between animal models and humans, it could be that compound selectivity for human proteins is actually not represented in animal models.

\section{Discussion}

Here, we present two CRISPR/Cas9 generated knockout zebrafish models for SCN1A-related epilepsies, including Dravet syndrome. The phenotype of Scn1Lab knockouts is characterized by spontaneous burst movements and sudden electrical discharges in the brain, a phenotype comparable to previous Scn1Lab knock-down models. Here, we used an optimized locomotor assay to extract burst movements from regular movement activity and found that the introduction of human SCN1A mRNA rescues this phenotype, indicating that ScnlLab and SCN1A have a comparable function. Scn1Lab knockouts show a reduction in whole organism GABA levels, and are more sensitive to convulsions induced by GABA antagonist PTZ, compared to wildtype larvae. These results mimic haploinsufficiency of SCN1A in humans that likely affect local GABA levels, leading to the seizure susceptibility in DS patients. By applying standard AEDs and DS specific drugs we observed a comparable pharmacological response as in DS patients, but also highlighted differences in drug response in comparison to previous Scn1Lab knock-down models. Finally, we show that the $\mathrm{Na}_{\mathrm{V}} 1.1$ activator AA43279 
and $\mathrm{Na}_{\mathrm{V}} 1.6$ selective compounds reduced the burst movement phenotype in Scn1Lab knockouts, indicating that selective ion channel inhibition or activation could be beneficial in epilepsy.

In our study, the non-selective VGSC activator Veratridine induced burst movements in wildtype zebrafish confirming its convulsing properties and suggesting that it likely has stronger effects on Nav1.6 inducing seizures, rather than increasing inhibition through Nav1.1. However, there was no effect in the ScnlLab knockouts, underlining that treatment of SCN1A haploinsufficiency could benefit from $\mathrm{Na}_{\mathrm{V}}$ activators and the need for VGSC subtype selective compounds. AA43279 is a small molecule with reasonable selectivity for $\mathrm{Na}_{\mathrm{V}} 1.1$ over the other $\mathrm{Na}_{\mathrm{V}}$ subtypes and showed a seizure reducing effect in our ScnlLab knockouts. However, with lesser affinity AA43279 could also activate other $\mathrm{Na}_{\mathrm{V}}$ subtypes (off-target activation), potentially leading to unwanted side effects. For example, off-target activation could lead to myotonia $\left(\mathrm{Na}_{\mathrm{V}} 1.4\right)$, atrial fibrillation and possible cardiac arrest $\left(\mathrm{Na}_{\mathrm{V}} 1.5\right)$, seizures $\left(\mathrm{Na}_{\mathrm{V}} 1.2\right)$ or painful neuropathy $\left(\mathrm{Na}_{\mathrm{V}} 1.7\right.$ and $\left.\mathrm{Na}_{\mathrm{V}} 1.8\right)$. For this reason, only compounds with high selectivity and efficacy at a very low dose are suitable candidates for translation to human patients. On the other hand, with future improved drug delivery systems such as nanoparticle liposomes targeting the CNS specifically, off-target activation in the peripheral nervous system could be limited.

Inhibition, rather than activation of VGSC is a treatment method that might be preferential, especially when inhibition can be targeted to one or few channel subtypes only. Inhibitors of VGSC have been used for decades in epilepsy patients and although current VGSC blocking drugs such as carbamazepine and phenytoin are hardly selective, they have been proven safe in humans. Therefore, to assess whether inhibition of Nav1.6 can be a novel treatment strategy for DS, also applicable for epilepsy caused by SCN8A gain-of function mutations and perhaps also epilepsy in general, we tested two compounds that selectively inhibit $\mathrm{Na}_{\mathrm{V}} 1.6$ 
channels. MV1312 showed a seizure reducing effect in our Scn1Lab knockouts comparable to Fenfluramine and other DS specific drugs. For this reason, we believe that $\mathrm{Na}_{\mathrm{V}} 1.6$ selective inhibitors could be just as efficient as $\mathrm{Na}_{\mathrm{V}} 1.1$ selective activators and are potentially a safer choice in human patients. Another compound, MV1369 was found to be selective for $\mathrm{Na}_{\mathrm{V}} 1.6$ over $\mathrm{Na}_{\mathrm{V}} 1.2$ and reduces the locomotor phenotype in Scn1Lab knockouts as well. There are several reasons why this type of compound is equally effective in the ScnlLab knockout. First, compounds that are selective for human ion channel proteins, could have a different effect when applied to animal models, as the proteins are not identical to those in humans. Only minor differences on nucleotide level could allow, or limit proper binding of compounds in the protein binding site. Second, not all VGSC genes are evolutionary conserved in zebrafish, but only 6 other ion channel genes exist beside Scn1Laa and Scn1Lab: Scn4aa/ab, Scn5Laa/ab and Scn8aa/ab ${ }^{[25]}$. While the function of these genes is not yet fully understood, it is clear that the absence of the full spectrum of $\mathrm{Na}_{\mathrm{V}}$ subtypes in zebrafish limits measurable side-effects. As Scn1Lab is knocked out in our model, the supposed remaining inhibitory neurotransmission is regulated by Scn1Laa, highlighted by the efficacy of the $\mathrm{Na}_{\mathrm{V}} 1.1$ activator AA43279. However, it could very well be that Scn1Laa is not a complete functional duplicant of Scn1Lab, but carries a shared function, and protein structure of $S C N 1 A, S C N 2 A$, $S C N 3 A$ and even $S C N 9 A$ as proposed earlier ${ }^{[25]}$. Therefore, efficacy, safety and administration routes of compounds tested in zebrafish, should be further studied in a human model system, with human genes and proteins. To further improve treatment of genetic epilepsy, and reach selective activation or inhibition with more potency and selectivity, drugs should be designed that act on the nucleotide level. As disorders such as DS have a genetic cause, their treatment should also act at the genome-or transcriptome level. For this reason we are looking forward to clinically relevant treatments based on small RNAs, CRISPRi and others that act on the SCN1A or SCN8A gene directly. 


\section{Materials \& Methods}

\section{Zebrafish maintenance \& ethics statement}

Adult zebrafish stocks of TL strain (Tubingen longfin) were maintained at $28.0^{\circ} \mathrm{C}$ on a $14 / 10$ hour light/dark cycle under standard aquaculture conditions. Embryos were kept under constant light condition in embryo medium (E3); $5 \mathrm{mM} \mathrm{NaCl}, 0.17 \mathrm{mM} \mathrm{KCl}, 0.33 \mathrm{mM}$ $\mathrm{CaCl} 2$, and $0.33 \mathrm{mM} \mathrm{MgSO} 4)$ at $28.5^{\circ} \mathrm{C}$. For all experiments described, larvae of $5 \mathrm{dpf}$ were used. All zebrafish experiments carried out were approved by the Animal Experimentation Committee of the Royal Netherlands Academy of Arts and Sciences. For imaging, larvae were embedded in 2\% low-melting point agarose prepared with E3 medium.

\section{sgRNA design and Cas9 preparation}

Gene specific guide RNAs (sgRNAs) were designed targeting Scn1Lab exon 10, using CHOPCHOP ${ }^{[29]}$ with an off-target binding cut-off of 4 or more base pair mismatches. sgRNA oligonucleotides were synthesized according to previously described methods ${ }^{[30]}$. Oligos are listed in S1 Table. Capped Cas9 mRNA was created by in vitro transcription using Thermo Fisher mMESSAGE mMACHINETM SP6 Transcription Kit from pCS2-nls-zCas9-nls (Addgene\#47929)

\section{CRISPR/Cas9-sgRNA injections and genotyping}

Fertilized eggs were injected with $2 \mathrm{nl}$ of a solution containing 500ng Cas 9 mRNA, 150ng sgRNA and 0.2uL Phenol Red. sgRNAs targeting efficiency was tested by PCR amplifying the target region of 8 injected eggs on $2 \mathrm{dpf}$. Primer sequences used for genotyping are listed in S1 Table. Injected embryos were raised to adult mosaic fishes. F0 founders were identified from week 10 by genotyping. F0 founders were outcrossed with wildtype fish to generate F1 embryos. F1 embryos were sampled for genotyping to confirm germline transmission of the mutation. The remaining F1 embryos were raised to adulthood and genotyped at week 10 by 
fin-clipping. Heterozygous knockouts carrying the same mutations were selected and crossed to raise the homozygous knockout F2 generation.

\section{CRISPR off-target assay}

The gene-specific region including the protospacer adjacent motif (PAM) of the sgRNA was submitted to CCtop ${ }^{[31]}$ to detect potential off-target sites. Five potential off-target sites with a maximum of four mismatches were selected for Sanger sequencing. Target sites, locations and the primers used for sequencing are included in S1 Table.

\section{GABA measurements}

Scn1Lab knockout or wildtype larvae were pooled $(\mathrm{n}=20)$ in eppendorf tubes in triplicates. Samples were centrifuged at $3500 \mathrm{rpm}$ for 12 minutes at $4 \mathrm{C}^{\circ}$ after which they were lysed in $500 \mu \mathrm{L}$ pre-chilled methanol using $0,5 \mathrm{~mm}$ zirconium oxide beads in a bullet blender. Samples were diluted 10 times and frozen at $-80 \mathrm{C}^{\circ}$ until day of analysis. A detailed Mass-spectrometry procedure be found in the Supplementary data.

\section{Locomotor assay}

Locomotor experiments were performed under dark conditions on $5 \mathrm{dpf}$ embryos placed in a flat bottom 48 -well cell culture dish filled with $1 \mathrm{~mL}$ E3/drug solution. Larvae were placed in single wells at 4 dpf to prevent stress from pipetting on the day of measurement. Movements were tracked in an automated tracking device (ZebraBox ${ }^{\mathrm{TM}}$ apparatus; Viewpoint, Lyon, France) for 90 minutes, stacked in 10 minute bins, of which the first 30 minutes were removed as habituation time for the locomotor chamber. The final recording time for all locomotor experiments yielded one hour total. Threshold parameters of viewpoint software were freezing 1 , sensitivity 8 , burst 50 resulting in a burst movement cut-off value of $>50 \mathrm{~mm} / \mathrm{s}$. Locomotor activity was quantified and analyzed by ZebraLab TM software (Viewpoint).

\section{hSCN1A Rescue}


The SCN1A plasmid, which encodes the human neonatal Nav1.1 ion channel, was previously described [28][32]. Capped hSCN1A mRNA was in vitro transcribed using Thermo Fisher mMESSAGE mMACHINETM T3 Transcription Kit. For mRNA injections, 200ng/ul mRNA was injected in the yolk of one-cell stage zebrafish embryos.

\section{Local field potential recordings}

The constructed LFP setup and concurrent recording settings were based on previous work [26] with slight modifications. In short, a glass electrode, connected to a high-impedance amplifier, was filled with $1 \mathrm{mM} \mathrm{NaCl}$. A larva was then embedded in $2 \%$ low-melting-point agarose (Invitrogen) and the glass electrode (2-7 M 2 ) placed on top of the forebrain of the larva. The recordings were performed in current clamp mode using the DAGAN EX-1 amplifier, national instruments 6210 USB digitizer and WinEDR software. The following parameters were used: low-pass filtered at $3 \mathrm{kHz}$, high-pass filtered $0.3 \mathrm{~Hz}$, digital gain 10 and sampling interval $10 \mu$ s. Single recordings were performed for ten minutes.

\section{Synthesis of MV1312 (3) and MV1369 (6)}

Synthesis of MV1312 (4-Chloro-n-\{3-[2-(4-methoxy-phenyl)-1h-imidazol-4-YL]-phenyl\}benzamide) and MV1369 (2-(3-methoxy-phenyl)-5-methyl-4-propyl-1h-imidazole) is depicted in Scheme 1. Details on the synthesis procedure can be found in the Supplementary data.<smiles>O=CC(=O)c1cccc(NC(=O)c2ccc(Cl)cc2)c1</smiles>

1<smiles>CCCC(=O)C(C)=O</smiles>
4<smiles>COc1ccc(C=O)cc1</smiles>

2

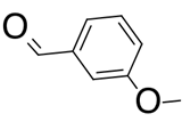

5<smiles>COc1ccc(-c2nc(-c3cccc(NC(=O)c4ccc(Cl)cc4)c3)c[nH]2)cc1</smiles>

3

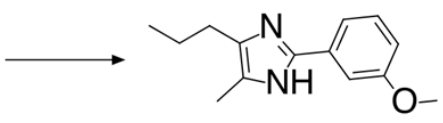

6

Scheme 1. Reagents and conditions: 1 equiv of 2 and 5 with 4 equiv $\mathrm{CH}_{3} \mathrm{COONH}_{4}$ in $3.8 \mathrm{~mL}$ 
$\mathrm{CH}_{3} \mathrm{OH}$; 1 equiv of 1 and 4 (respectively) in $3.5 \mathrm{~mL} \mathrm{CH} \mathrm{CH}_{3} \mathrm{OH}$. Overnight rt.

\section{Determination of compound selectivity}

Activity of MV1312 and MV1369 at hNav1.1-1.8 was assessed using a fluorescent imaging plate reader (FLIPRTetra, molecular devices) membrane potential assay as previously described $^{55}$. In brief, cell lines (HEK293 Nav1.1-1.8) were plated on 384-well black-walled imaging plates (Corning) at a density of 10 000-15 0000 cells per well 48 hours before loading with $20 \mu \mathrm{L}$ of red membrane potential dye (proprietary formulation) (Molecular Devices, Sunnyvale, CA). Cells were incubated with the membrane potential dye for $30 \mathrm{~min}$ at $37^{\circ} \mathrm{C}$ before the addition of compounds by the FLIPR ${ }^{\text {Tetra }}$ system. After the addition of $100 \mu \mathrm{M}$ MV1312, fluorescence was measured (excitation 515-545 nm; emission: 565-625 $\mathrm{nm}$ ) for a period of $5 \mathrm{~min}$ to determine the effects of the compounds alone. Following this 5 min exposure, 5-20 $\mu \mathrm{M}$ VRT was added and fluorescence was measured for a further 5 min. Data was recorded and converted to response over baseline using Screenworks 3.2.0.14.

\section{Drug treatment}

E3/drug solutions (2X) were prepared in $0.8 \%$ DMSO at their maximum tolerated

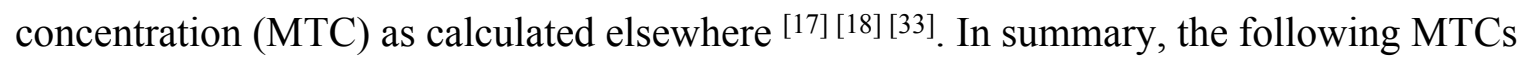

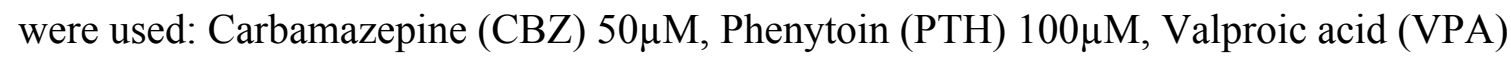
$100 \mu \mathrm{M}$, Stiripentol (STP) $12,5 \mu \mathrm{M}$, Fenfluramine (FA) $50 \mu \mathrm{M}$, Clemizole (CLM) $100 \mu \mathrm{M}$. $500 \mu \mathrm{L}$ of AED solution was pipetted in each single well containing $500 \mu \mathrm{L}$ E3 generating a final DMSO concentration of $0.4 \%$. For short incubation experiments embryos were incubated for 1 hour, for long incubation experiments, the incubation time was 18 hours. Compounds were ordered via Sigma-Aldrich.

\section{Determination of maximum tolerated concentration (MTC)}

Compounds AA43279, MV1312 and MV1369 were tested for toxicity based on previously established methods ${ }^{[17]}$ with slight modifications. In short; compounds were incubated in the 
bathing medium of $4 \mathrm{dpf}$ larvae. After 24 hours, the following toxicity parameters were checked: touch response, loss of posture, body deformation and death. When none of these parameters were observed in any of the larvae tested, the concentration was regarded safe (data not shown).

\section{Statistical analysis}

Data was analyzed and plotted using Graphpad Prism 7.04. Locomotor data did not pass the D'agostino \& Pearson normality test, therefore the non-parametric Mann-Whitney U-test was used for data analysis. Mass-spectrometry was normally distributed and was further analyzed using the student t-test. Compound selectivity data was normally distributed and therefore analyzed with a multi-comparison ANOVA. A $P$-value of $<0.05$ was considered significant.

\section{Acknowledgement}

The authors would like to thank Prof. Dr. J. Bakkers and Dr. F. Tessadori (Hubrecht Institute Utrecht, the Netherlands) for help and advice during the project. Compound AA43279 was a kind gift of Dr. T. Benned-Jensen (H.Lundbeck A/S Copenhagen). The authors would like to thank Prof. P. de Witte (K.U. Leuven, Belgium) and Dr. A. Zdebik (U.C. Londen, England) for their advice on the electrophysiology setup.

\section{Author contributions}

Conceived project: BPCK KPJB. Designed and performed zebrafish experiments: WW SS MR RS MB NVD. Generated hSCN1A plasmid: LV. Designed and Performed compound synthesis and selectivity assays: MR MI IV. Analyzed the data and wrote the paper WW. MR. BPCK

\section{Conflict of interest}


Neither of the authors has any conflict of interest to disclose

\section{References}

1 Schoonjans AS, Lagae L, Ceulemans, B. Low-dose fenfluramine in the treatment of neurologic disorders: experience in Dravet syndrome Ther Adv Neurol Disord. $2015 ; 8: 328-338$

2 Parihar R, Ganesh S. The SCN1A gene variants and epileptic encephalopathies J Hum Genet. 2013;58: 573-80

3 Han S, Yu FH, Schwartz MD et al. NaV1.1 channels are critical for intercellular communication in the suprachiasmatic nucleus and for normal circadian rhythms Proc Natl Acad Sci U S A 2012; 109: E368-E377.

4 Lorincz A, Nusser Z. Cell-Type-Dependent Molecular Composition of the Axon Initial Segment Journal of Neuroscience 31 December 2008, 28 (53) 14329-14340

5 Depienne C, Trouillard O, Saint-Martin C, et al. Spectrum of SCN1A gene mutations associated with Dravet syndrome: analysis of 333 patients Journal of Medical Genetics 2009;46:183-191.

6 Meng H, Xu H, Yu L, et al. The SCN1A Mutation Database: Updating Information and Analysis of the Relationships among Genotype, Functional Alteration, and Phenotype Human mutation 2015; 36: 573-580

7 Ye M, Yang J, Tian C, et al. Differential roles of NaV1.2 and NaV1.6 in regulating neuronal excitability at febrile temperature and distinct contributions to febrile seizures Sci Rep. 2018;8:753. 
8 Møller RS, Johannesen KM, Precision Medicine: SCN8A Encephalopathy Treated with Sodium Channel Blockers Neurotherapeutics 2016;13:190-191

9 Boerma RS, Braun KP, van den Broek MP, et al. Remarkable Phenytoin Sensitivity in 4 Children with SCN8A-related Epilepsy: A Molecular Neuropharmacological Approach. Neurotherapeutics 2016; 13:192-7

10 Wang J, Gao H, Bao X, et al. SCN8A mutations in Chinese patients with early onset epileptic encephalopathy and benign infantile seizures BMC Med Genet. 2017; 18: 104.

11 Wirrell EC. Treatment of Dravet Syndrome. Can J Neurol Sci. 201643 Suppl 3 S13-

12 Richards KL, Milligan CJ, Richardson RJ, et al. Selective NaV1.1 activation rescues Dravet syndrome mice from seizures and premature death PNAS 2018 115:E8077E8085

13 Escoubas P, Diochot S, Célérier M, et al. Novel Tarantula Toxins for Subtypes of Voltage-Dependent Potassium Channels in the Kv2 and Kv4 Subfamilies Mol Pharmacol 2002; 62:48-57

14 Martin MS, Tang B, Papale, LA, et al. The voltage-gated sodium channel Scn8a is a genetic modifier of severe myoclonic epilepsy of infancy. Human molecular genetics 2007; 16: 2892-9

15 Frederiksen K, Lu D, Yang J, et al. A small molecule activator of Nav 1.1 channels increases fast-spiking interneuron excitability and GABAergic transmission in vitro and has anti-convulsive effects in vivo. Eur J Neurosci. 2017;46:1887-1896 
16 Rosker C, Lohberger B, Hofer D, et al. The TTX metabolite 4,9-anhydro-TTX is a highly specific blocker of the $\mathrm{Na}(\mathrm{v} 1.6)$ voltage-dependent sodium channel. Am J Physiol Cell Physiol. 2007; 293:C783-9.

17 Zhang Y, Kecskés A, Copmans D, et al. Pharmacological Characterization of an Antisense Knockdown Zebrafish Model of Dravet Syndrome: Inhibition of Epileptic Seizures by the Serotonin Agonist Fenfluramine PLoS One 2015; 10: e0125898.

18 Baraban SC1, Dinday MT, Hortopan GA. Drug screening in Scn1a zebrafish mutant identifies clemizole as a potential Dravet syndrome treatment. Nat Commun. $2013 ; 4: 2410$

19 Griffin A, Hamling KR, Knupp K, et al. Clemizole and modulators of serotonin signalling suppress seizures in Dravet syndrome Brain 2017 140: 669-683.

20 Eimon PM, Ghannad-Rezaie M, De Rienzo G, et al. Brain activity patterns in highthroughput electrophysiology screen predict both drug efficacies and side effects Nature Communications 2018;9:219

21 de Bruijn E1, Cuppen E, Feitsma H. Highly Efficient ENU Mutagenesis in Zebrafish Methods Mol Biol. 2009;546:3-12

22 Chen T, Wang Y, Wu Y. Developmental exposures to ethanol or dimethylsulfoxide at low concentrations alter locomotor activity in larval zebrafish: Implications for behavioral toxicity bioassays Aquatic Toxicology 2011 102: 162-166

23 El-Brolosy MA, Stainier DYR. Genetic compensation: A phenomenon in search of mechanisms Plos Genetics 2017+ 13: e1006780.

24 Lawson ND. Reverse Genetics in Zebrafish: Mutants, Morphants, and Moving Forward Trends in Cell Biology 2016 26: 77-79 
25 Novak AE, Taylor AD, Pineda RH, et al. Embryonic and larval expression of zebrafish voltage-gated sodium channel alpha-subunit genes. Dev Dyn. 2006;

235: $1962-73$

26 De Witte P, Lagae, L. Serotonergic modulation as a pharmacological modality in the treatment of Dravet syndrome Brain 2017; 140: e35

27 Kantae V, Krekels EH, Ordas A, et al. Pharmacokinetic Modeling of Paracetamol Uptake and Clearance in Zebrafish Larvae: Expanding the Allometric Scale in Vertebrates with Five Orders of Magnitude Zebrafish 2016 13:504-510

28 Hawkins NA, Anderson LL, Gertler TS, et al. Screening of conventional anticonvulsants in a genetic mouse model of epilepsy. Ann Clin Transl Neurol. 2017 4:326-339

29 Labun K, Montague TG, Gagnon JA, et al; CHOPCHOP v2: a web tool for the next generation of CRISPR genome engineering. Nucleic Acids Research 2016; 44: W2726

30 Gagnon JA, Valen E, Thyme SB, et al. Efficient Mutagenesis by Cas9 ProteinMediated Oligonucleotide Insertion and Large-Scale Assessment of Single-Guide RNAs PLoS ONE 2014; 9: e98186

31 CCtop: Stemmer M., Thumberger T, del Sol Keyer, M., et al. CCTop: an intuitive, flexible and reliable CRISPR/Cas9 target prediction tool. PLOS ONE 2015; 10: $\mathrm{e} 0124633$

32 Volkers L, Kahlig KM, Verbeek NE, Nav1.1 dysfunction in genetic epilepsy with febrile seizures-plus or Dravet syndrome European Journal of Neuroscience 2011; 34: $1268-1275$ 
33 Afrikanova T, Serruys AK, Buenafe OEM, Validation of the Zebrafish

Pentylenetetrazol Seizure Model: Locomotor versus Electrographic Responses to Antiepileptic Drugs PLoS One 2013; 8: e54166

34 Deuis JR, Dekan Z, Inserra MC, et al. Development of a muO-Conotoxin Analogue with Improved Lipid Membrane Interactions and Potency for the Analgesic Sodium Channel NaV1.8. The Journal of biological chemistry 2016:291; 11829-11842

35 Tomson T, Battino D, Perucca E. Valproic acid after five decades of use in epilepsy: time to reconsider the indications of a time-honoured drug The Lancet Neurology $2016 ; 15 ; 210-218$

36 Ruffolo G, Cifelli P, Roseti C. A novel GABAergic dysfunction in human Dravet syndrome Epilepsia 2018; 59: 2106-2117

37 Braat S, Kooy F. The GABAA Receptor as a Therapeutic Target for Neurodevelopmental Disorders Neuron 2015;86:1119-1130

38 Qiao X, Sun G, Clare JJ. Properties of human brain sodium channel $\alpha$-subunits expressed in HEK293 cells and their modulation by carbamazepine, phenytoin and lamotrigine Br J Pharmacol. 2014:171; 1054-1067.

39 Cardenas CA1, Cardenas CG, de Armendi AJ, et al. Carbamazepine interacts with a slow inactivation state of NaV1.8-like sodium channels Neurosci Lett. 2006: 408; 129-34.

40 Yu FH, Mantegazza M, Westenbroek RE, et al. Reduced sodium current in GABAergic interneurons in a mouse model of severe myoclonic epilepsy in infancy Nature neuroscience 2006;9:1142-1149 
41 Escayg A, and Goldin AL. Sodium channel SCN1A and epilepsy: mutations and mechanisms Epilepsia 2010; 51:1650-1658.

\section{Figure legends}

Figure 1 Morphology of Scn1Lab knockout. A) 5 dpf Scn1Lab homozygous knockout larvae show hyperpigmentation and the absence of an inflated swim-bladder. These morphological defects are absent in heterozygous (B) or wildtype (C) zebrafish larvae.

Figure 2 A) Spontaneous burst movements quantified in two knockout lines using the locomotor burst movement assay B) Burst movements are unique to scn1lab knockouts, and not caused by the absence of an inflated swimming bladder C) The burst movement phenotype of scn1lab knockouts is partially rescued when human SCN1A, either mRNA or cDNA is introduced D) Scn1Lab knockouts show a reduction in free GABA levels E) Both heterozygote and homozygous Scn1lab knockouts show sensitivity to exposure of 5mM pentylenetetrazole F) Representative non-invasive local field potential recordings from the brain of wildtype and Scn1Lab knockout zebrafish (n=3). Scn1Lab knockout zebrafish show spontaneous electrical discharges that resemble seizure activity. Error bars $=$ S.D. $(-/-)$ $=$ Scn1Lab knockout, locomotor assay $n=12$ per group

Figure 3 Pharmacologic validation of Scn1Lab knockouts by short (60 minutes) or long (18 hours) exposure of anti-epileptic drugs to the swimming medium A) $50 \mu \mathrm{M}$ Carbamazepine B) $100 \mu \mathrm{M}$ Phenytoin C) $100 \mu \mathrm{M}$ Sodium valproate D) $12.5 \mu \mathrm{M}$ Stiripentol E) $50 \mu \mathrm{M}$ Fenfluramine F) $50 \mu \mathrm{M}$ Clemizole. Dashed lines indicate a novel experimental plate with a seperate experimental group. Error bars $=$ S.D., (-/-) = ScnlLab knockout, $n=12$ per group.

Figure 4 The effect of VGSC activators on burst movements in Scn1Lab knockouts A) $10 \mu \mathrm{M}$ Veratridine B) 5uM AA43279 Error bars $=$ S.D. $(-/-)=$ Scn1Lab knockout, $n=12$ per group .

Figure 5 Selectivity of ion channel blocking compounds A) MV1312 show blocking selectivity for $\mathrm{Na}_{\mathrm{V}} 1.6$ over the other ion channel subtypes but not $\mathrm{Na}_{\mathrm{V}} 1.8$. B) The effect of $5 \mu \mathrm{M} \mathrm{MV} 1312$ on locomotor burst movements in Scn1Lab knockout zebrafish C) MV1369 shows blocking selectivity 
bioRxiv preprint doi: https://doi.org/10.1101/675082; this version posted June 18,2019 . The copyright holder for this preprint (which was

not certified by peer review) is the author/funder, who has granted bioRxiv a license to display the preprint in perpetuity. It is made available under aCC-BY 4.0 International license.

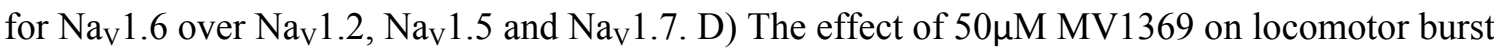
movements in Scn1Lab knockout zebrafish Error bars $=$ S.D., (-/-) $=$ Scn1Lab knockout, locomotor assays $n=12$ per group, selectivity assays $n=3$ per group, $*<0.05 * *<0.005 * * *<0.0005$. 
A
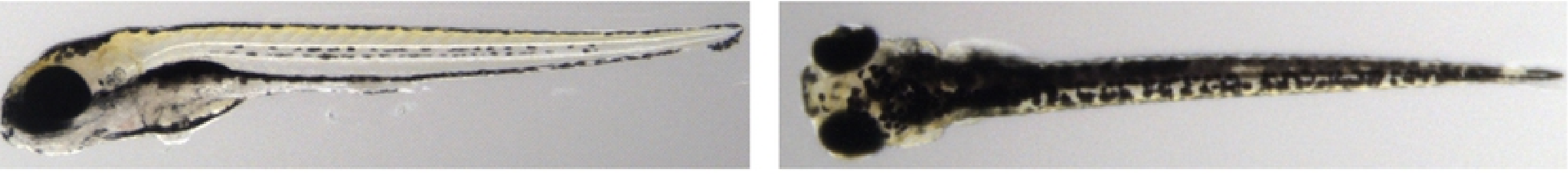

B
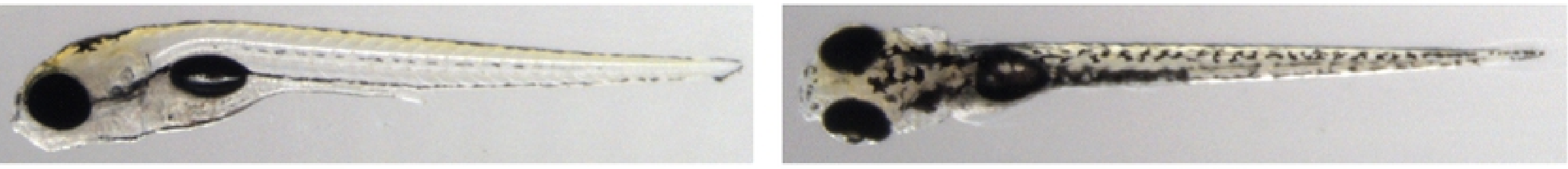

C
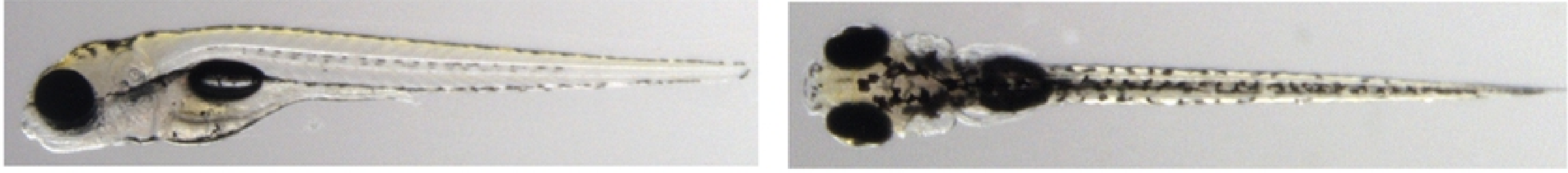

Figure 1 
A Spontaneous burst movements B nsb control
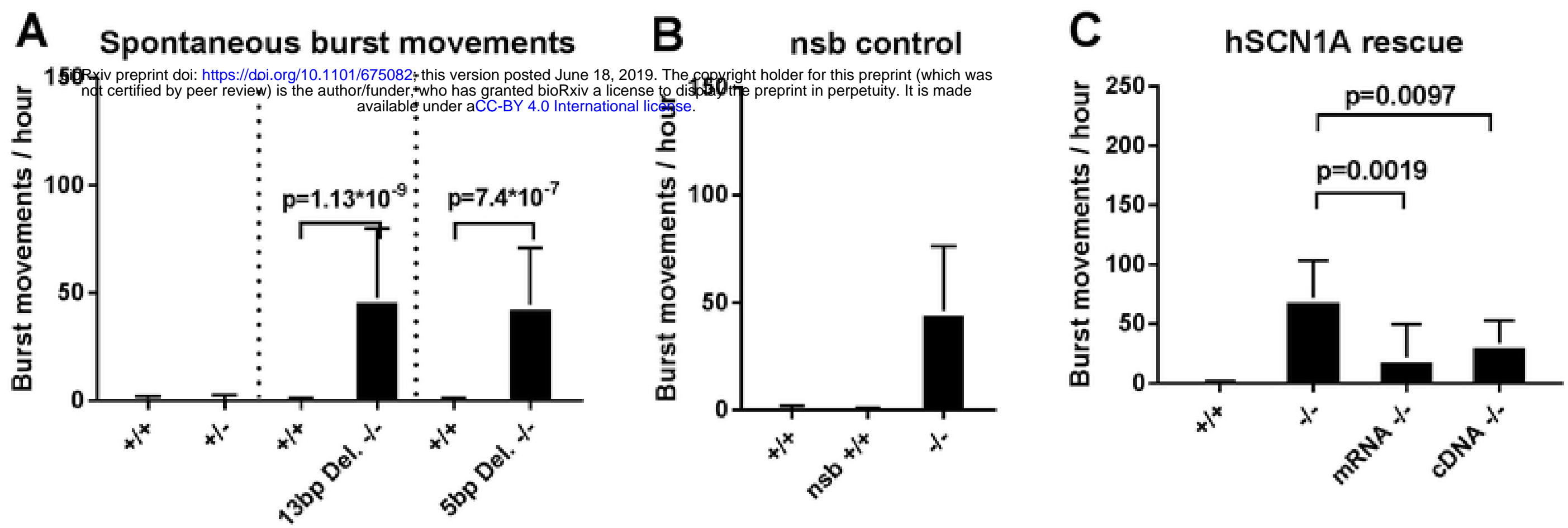

D

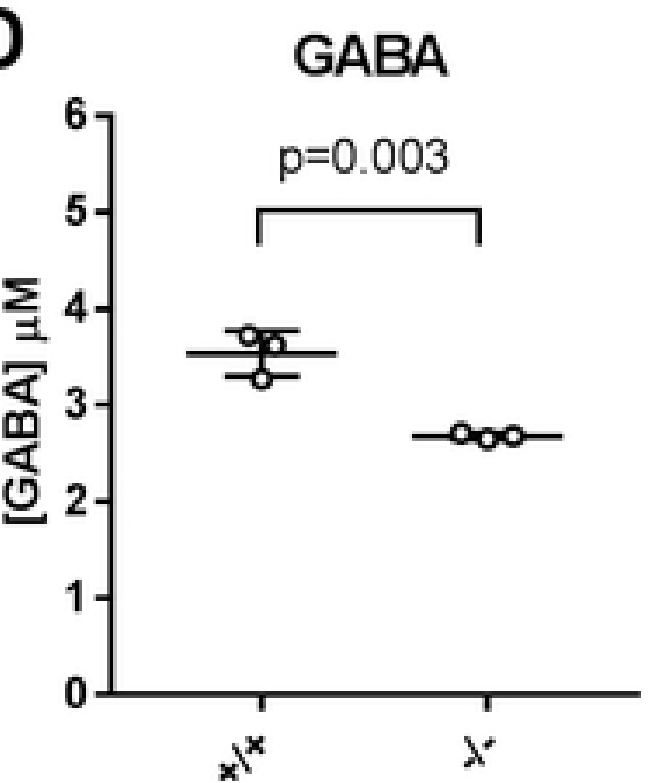

E Pentylenetetrazole sensitivity

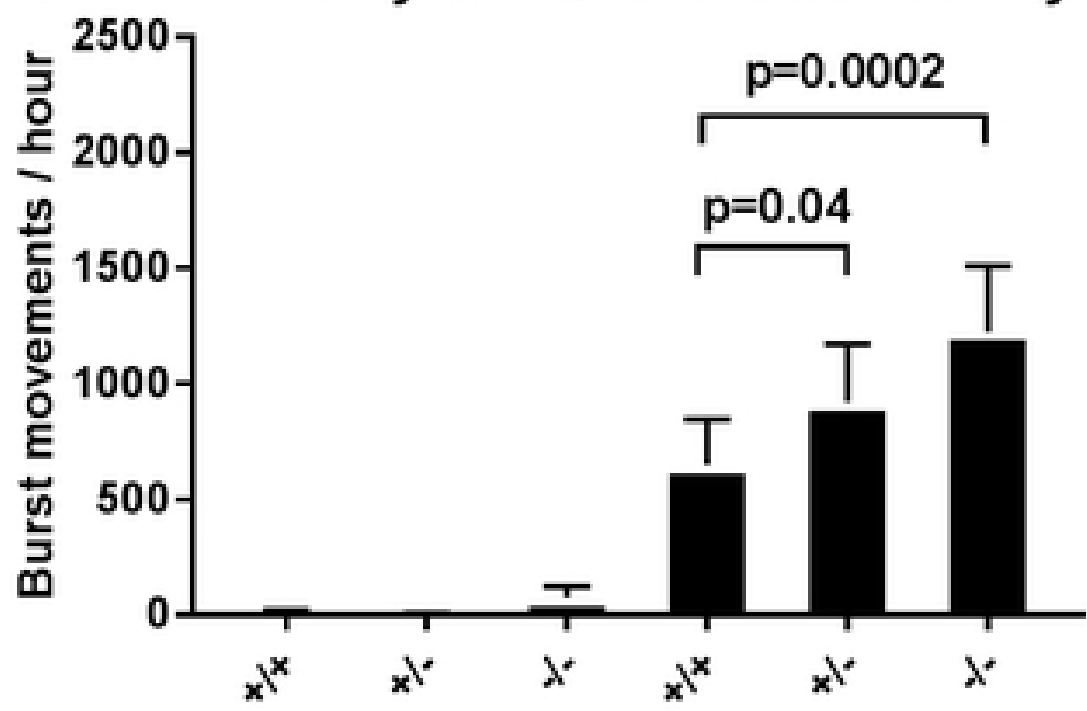

untreated

PTZ

F Local field potential recording of the brain

Wildtype

Scn1Lab knockout

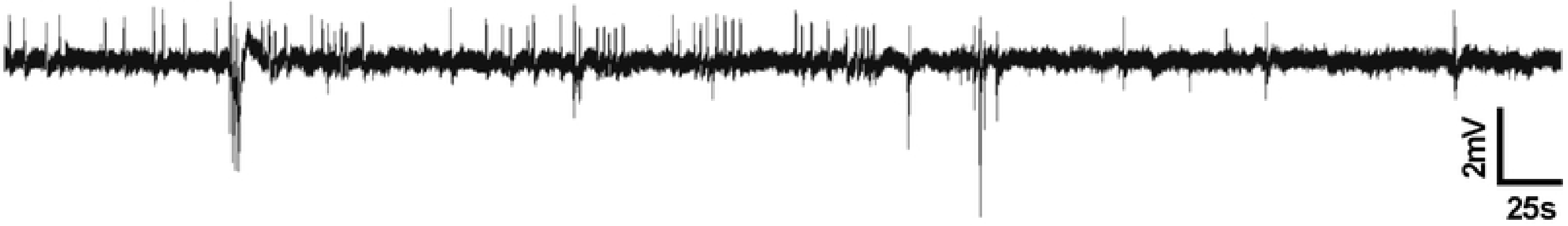

Figure 2 
A

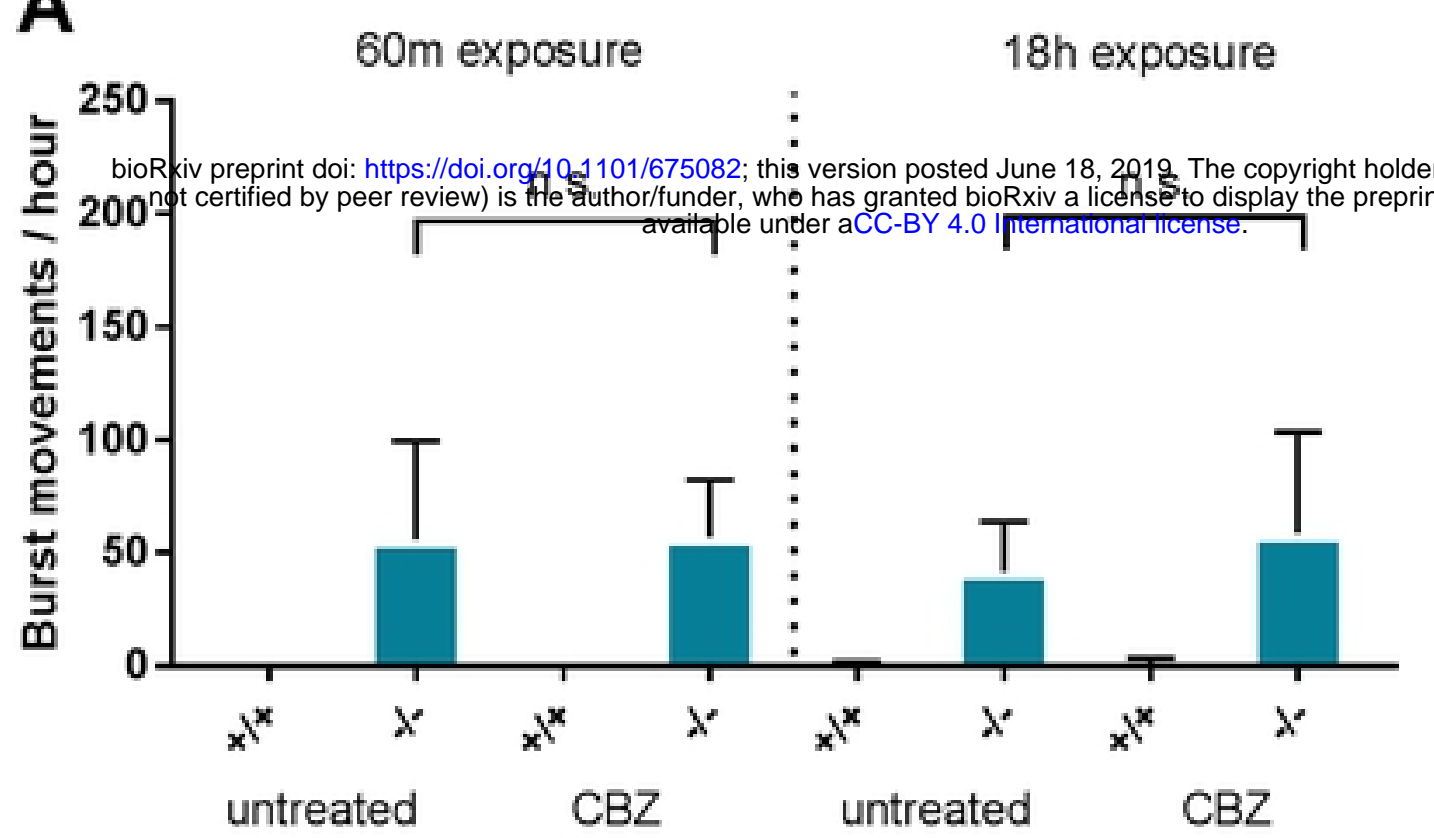

B
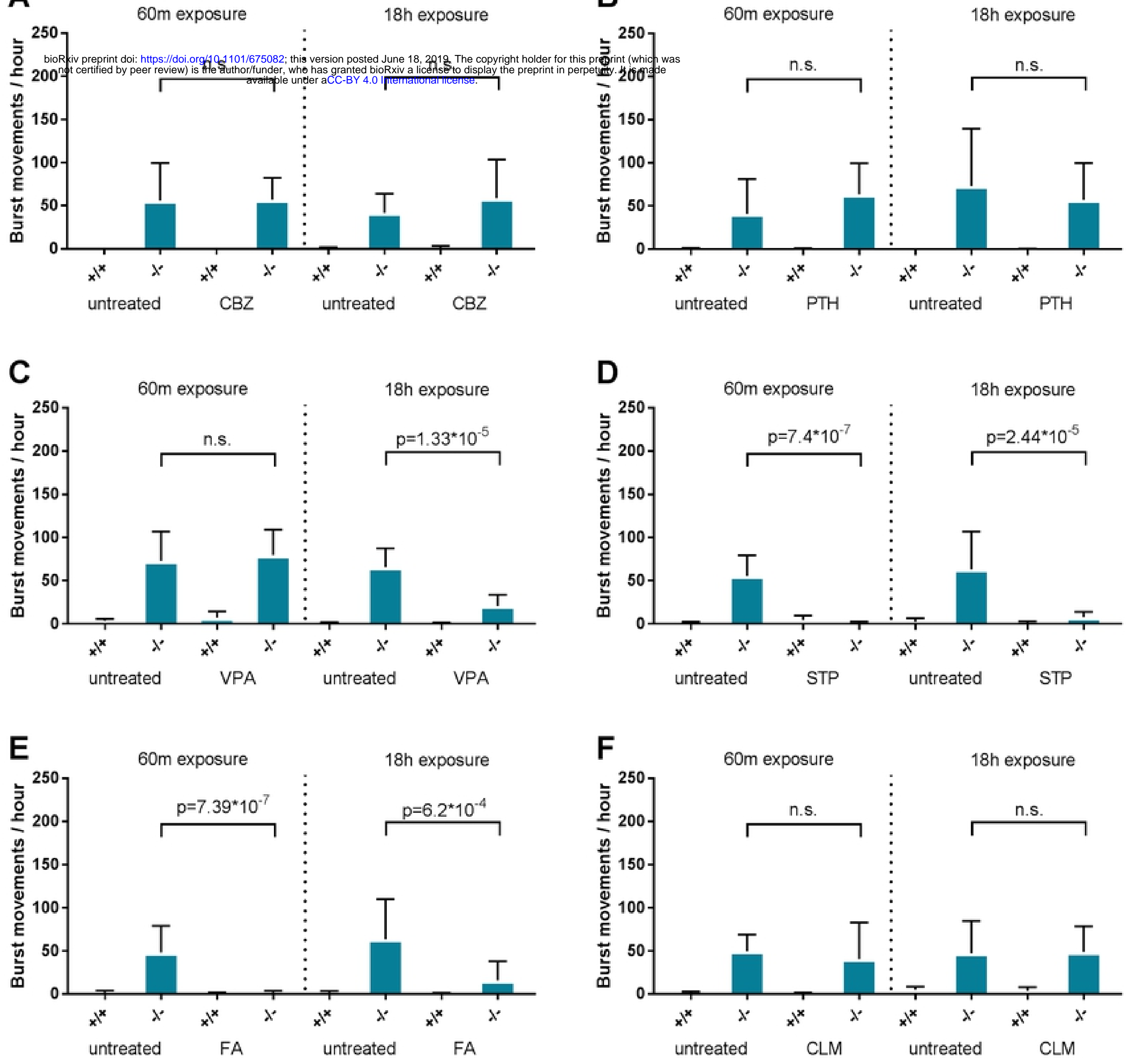

Figure 3 


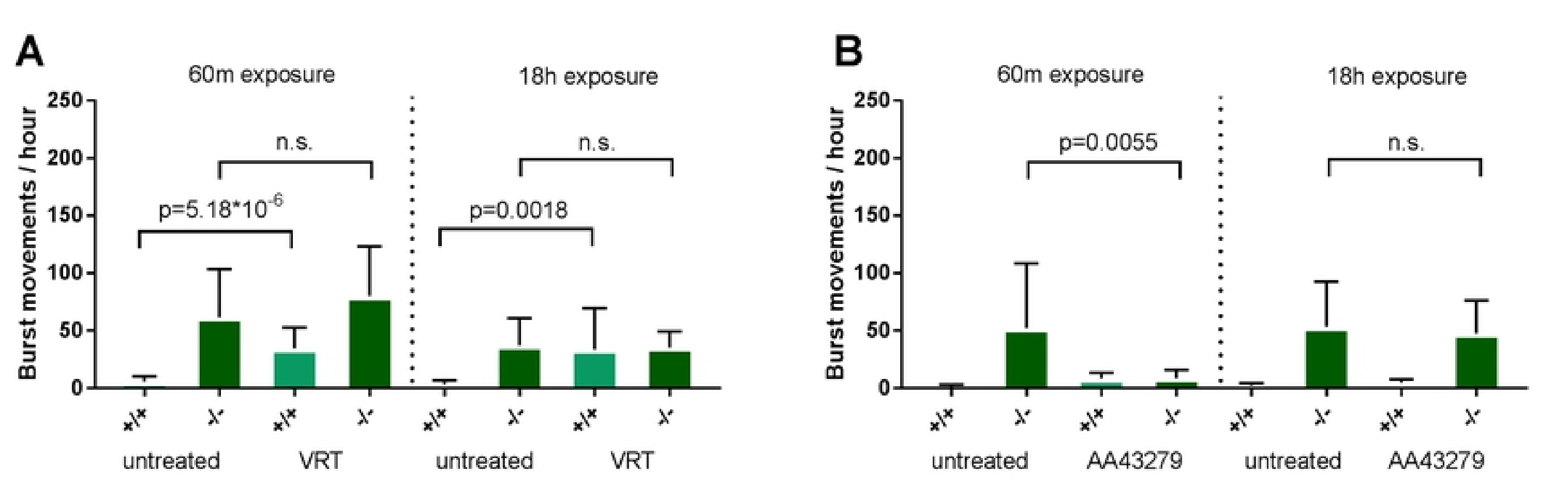

Figure 4 
A

MV1312 selectivity
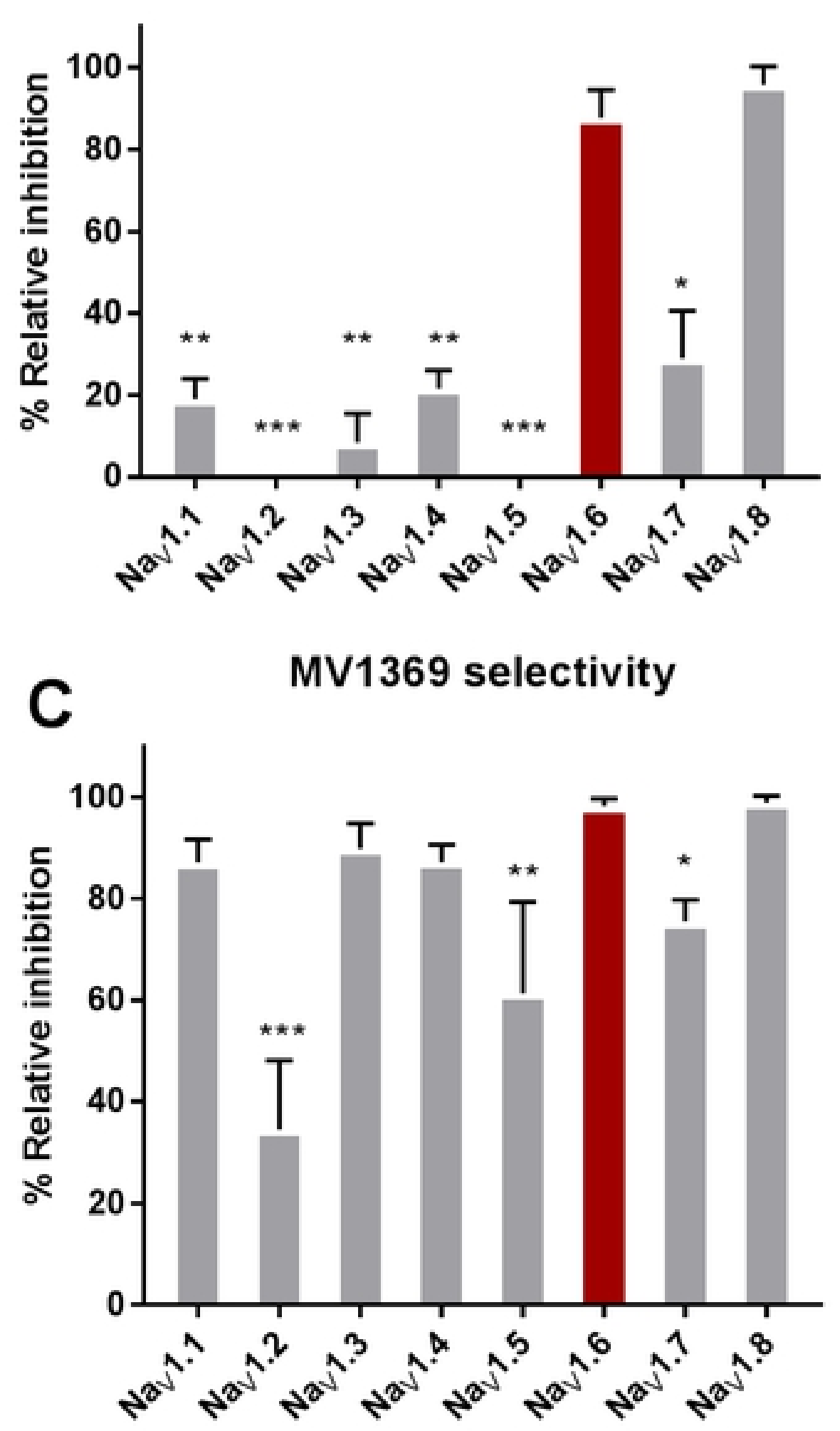

B

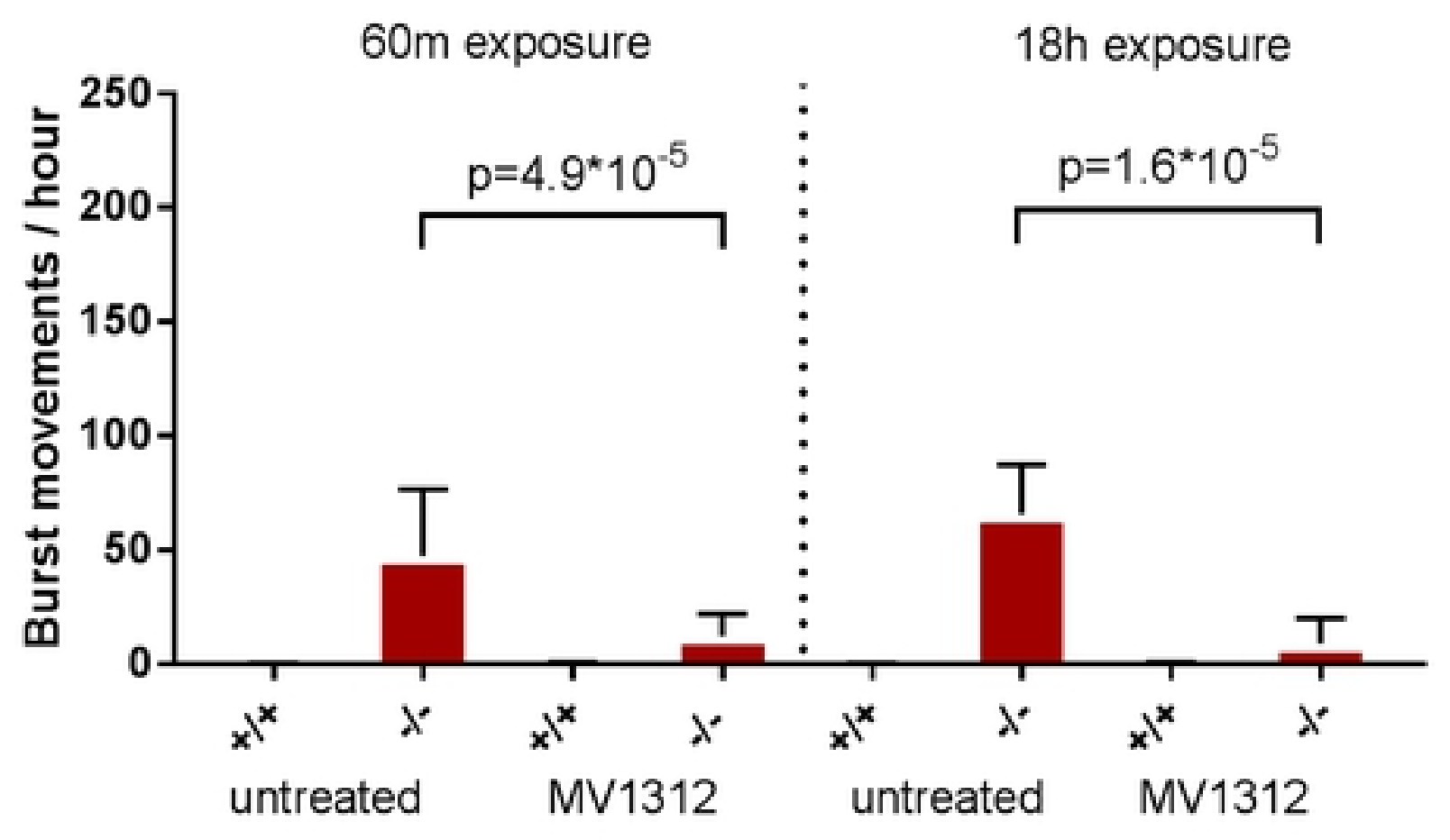

D

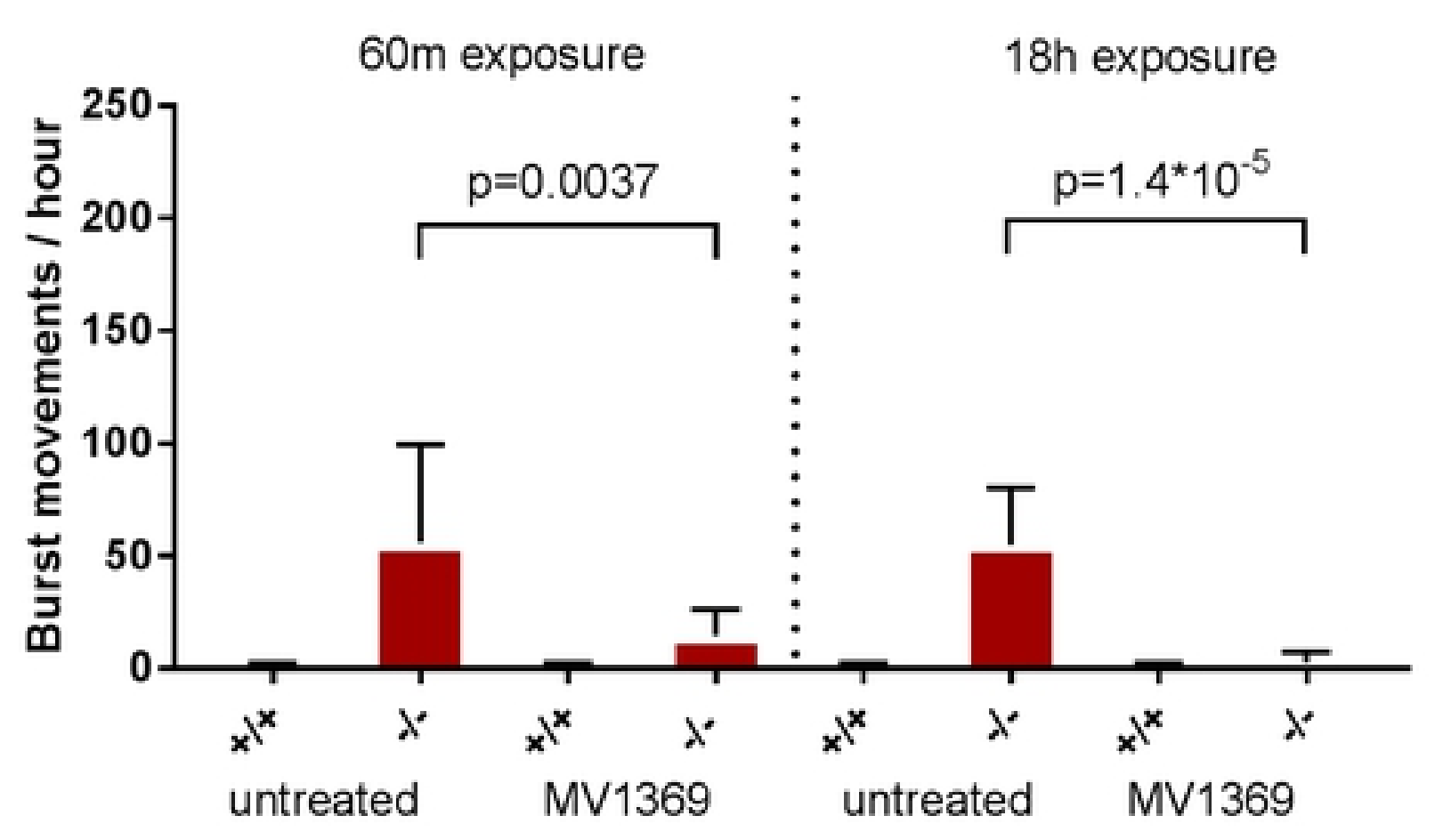

Figure 5 